\title{
Energy-Aware Memory Mapping for Hybrid FRAM-SRAM MCUs in Intermittently-Powered loT Devices
}

\author{
HRISHIKESH JAYAKUMAR, ARNAB RAHA, JACOB R. STEVENS, \\ and VIJAY RAGHUNATHAN, Purdue University
}

Forecasts project that by 2020, there will be around 50 billion devices connected to the Internet of Things (IoT), most of which will operate untethered and unplugged. While environmental energy harvesting is a promising solution to power these IoT edge devices, it introduces new complexities due to the unreliable nature of ambient energy sources. In the presence of an unreliable power supply, frequent checkpointing of the system state becomes imperative, and recent research has proposed the concept of $i n$-situ checkpointing by using ferroelectric RAM (FRAM), an emerging non-volatile memory technology, as unified memory in these systems. Even though an entirely FRAM-based solution provides reliability, it is energy inefficient compared to SRAM due to the higher access latency of FRAM. On the other hand, an entirely SRAM-based solution is highly energy efficient but is unreliable in the face of power loss. This paper advocates an intermediate approach in hybrid FRAM-SRAM microcontrollers that involves judicious memory mapping of program sections to retain the reliability benefits provided by FRAM while performing almost as efficiently as an SRAM-based system. We propose an energy-aware memory mapping technique that maps different program sections to the hybrid FRAM-SRAM microcontroller such that energy consumption is minimized without sacrificing reliability. Our technique consists of $e M$-map, which performs a one-time characterization to find the optimal memory map for the functions that constitute a program and energy-align, a novel hardware-software technique that aligns the system's powered-on time intervals to function execution boundaries, which results in further improvements in energy efficiency and performance. Experimental results obtained using the MSP430FR5739 microcontroller demonstrate a significant performance improvement of up to $2 \mathrm{x}$ and energy reduction of up to $20 \%$ over a state-of-the-art FRAM-based solution. Finally, we present a case study that shows the implementation of our techniques in the context of a real IoT application.

CCS Concepts: • Computer systems organization $\rightarrow$ Embedded hardware; Embedded software; $\bullet$ Hardware $\rightarrow$ Emerging architectures;

Additional Key Words and Phrases: Intermittently powered systems, internet of things, energy harvesting, ferroelectric RAM, batteryless systems, checkpointing

ACM Reference Format:

Hrishikesh Jayakumar, Arnab Raha, Jacob R. Stevens, and Vijay Raghunathan. 2017. Energy-aware memory mapping for hybrid FRAM-SRAM MCUs in intermittently-powered IoT devices. ACM Trans. Embed. Comput. Syst. 16, 3, Article 65 (April 2017), 23 pages.

DOI: http://dx.doi.org/10.1145/2983628

\section{INTRODUCTION}

The advent of the Internet of Things (IoT) era has fueled the emergence of new applications that improve various aspects of everyday human life. An ever-increasing number

This work was supported in part by the National Science Foundation under grants CNS-0953468 and CCF1018358 and by the Semiconductor Research Corporation (SRC).

Authors' addresses: H. Jayakumar, A. Raha, J. R. Stevens, and V. Raghunathan, School of Electrical and Computer Engineering, Purdue University, West Lafayette, IN 47907; emails: \{hjayakum, araha, steven69, vr\}@purdue.edu; H. Jayakumar was affiliated with Purdue at the time the research reported in the manuscript was conducted. He has been affiliated with Qualcomm Inc. from Aug' 2016.

Permission to make digital or hard copies of part or all of this work for personal or classroom use is granted without fee provided that copies are not made or distributed for profit or commercial advantage and that copies show this notice on the first page or initial screen of a display along with the full citation. Copyrights for components of this work owned by others than ACM must be honored. Abstracting with credit is permitted. To copy otherwise, to republish, to post on servers, to redistribute to lists, or to use any component of this work in other works requires prior specific permission and/or a fee. Permissions may be requested from Publications Dept., ACM, Inc., 2 Penn Plaza, Suite 701, New York, NY 10121-0701 USA, fax +1 (212) 869-0481, or permissions@acm.org.

(c) 2017 ACM 1539-9087/2017/04-ART65 $\$ 15.00$

DOI: http://dx.doi.org/10.1145/2983628 
and types of IoT sensors (henceforth referred to as edge devices) are being deployed to seamlessly bridge the physical world with the world of the computing infrastructure. However, powering such deeply embedded IoT edge devices is extremely challenging due to their unique constraints such as remote deployment location, tiny form factor, and extreme longevity requirements [Jayakumar et al. 2014a, 2016b]. Environmental energy harvesting (where the system powers itself using energy that it scavenges from its operating environment) has been shown to be a promising and viable option for powering these IoT devices [Lu et al. 2010, 2011; Wang et al. 2014; Jayakumar et al. 2014b]. However, ambient energy sources (such as vibration, wind, radio frequency (RF) signals) are often unreliable and intermittent in nature, which can lead to frequent intervals of power loss [Bhatti et al. 2016]. Performing computations reliably in the face of such power supply interruptions is challenging and requires some form of checkpointing of the system state from SRAM to non-volatile memory when power loss is imminent. Traditionally, microcontrollers have employed flash memory as the primary non-volatile storage technology. However, the energy- (and latency-) intensive erase/write operations of flash make it inefficient for frequent checkpointing.

The emergence of non-volatile memory technologies such as ferroelectric RAM (FRAM), magnetoresistive RAM (MRAM), and resistive RAM (ReRAM), which have superior power and performance characteristics compared to flash memory, has led to new hybrid memory architectures. Low-power microcontrollers (MCUs) that integrate FRAM [Khanna et al. 2014; Wang et al. 2012], MRAM [Sakimura et al. 2014], and ReRAM [Liu et al. 2016] have already been demonstrated. Recent work [Jayakumar et al. 2015] has also shown that the use of FRAM as unified memory (where all program segments, including text, stack, data, and so on, are mapped to the FRAM) enables efficient in situ checkpointing in IoT devices, thereby allowing them to seamlessly perform long-running computations in the face of frequent power loss. Even though FRAM outperforms flash in terms of performance and power consumption, it is still inferior to SRAM due to inherent device limitations. For example, in TI's MSP430FR5739 [Texas Instruments 2014] microcontroller, accesses to FRAM are 3x slower and consume more energy as compared to SRAM. Therefore, executing programs from FRAM results in lower performance and higher energy consumption compared to executing programs from SRAM. On the other hand, an entirely SRAM-based solution is highly energy efficient when running continuously on reliable power but is unreliable in the face of power loss, because SRAM is volatile. This article advocates (and demonstrates the benefits of) an intermediate approach in hybrid FRAM-SRAM systems that involves judicious memory mapping of program sections to retain the reliability benefits provided by FRAM while performing almost as efficiently as an SRAM-based system, thus obtaining the best of both. Specifically, the article makes the following contributions:

-To the best of our knowledge, this is the first work to investigate energy-aware memory mapping for IoT devices that are based on hybrid FRAM-SRAM microcontrollers. We propose a comprehensive design methodology that synergistically combines the benefits of SRAM and FRAM technologies to efficiently, yet reliably, perform computations across power cycles in intermittently powered IoT systems.

-We propose a one-time characterization mechanism, eM-map, that determines the optimal memory mapping at the granularity of functions in a program. We also propose energy-align, a novel hardware-software technique that uses proactive system shutdown as a mechanism to align the time intervals when the system is powered on with function execution boundaries, which results in further improvements in energy efficiency, as we will demonstrate.

-We have implemented our memory-mapping technique on a custom hardware platform based on the Texas Instruments MSP430FR5739 MCU and have evaluated it 
using six typical benchmark applications used in IoT edge devices. Experimental results demonstrate performance improvements and energy savings of up to $2 \mathrm{x}$ and $20 \%$, respectively, compared to an existing state-of-the-art FRAM-only solution.

-We conduct a case study with a real IoT application that senses environmental conditions, performs computation on it, and then transmits the data wirelessly to an Android mobile phone using an experimental platform as a proof-of-concept for our technique.

\section{BACKGROUND AND RELATED WORK}

\subsection{Progressing Application Execution in Intermittently Powered loT Systems}

Enabling systems powered by unreliable and intermittent energy sources to perform long-running computations has been a topic of research in the recent past. Since systems with an unstable power supply may experience a power loss at any point in program execution, it is imperative to perform some sort of checkpointing of the system state before it shuts off. When the system receives power again, the saved snapshot is restored, thus enabling computations to execute across power cycles oblivious to the disruption in power supply. Giving applications this illusion of continuity is essential, without which programs could be stuck performing the same computations repeatedly in each power cycle without making any progress. A checkpoint operation done when the system detects that power loss is imminent seeks to provide this continuity. In such intermittently powered systems, a checkpoint operation can be triggered by comparing the energy remaining in the system with a pre-defined threshold. This threshold is determined by the total amount of energy that is required to complete a successful checkpoint $\left(E_{\text {ckpt }}\right)$, which is given by the equation below,

$$
E_{\text {ckpt }}=E_{\text {byte }} \times N_{\text {bytes }}
$$

where $E_{\text {byte }}$ indicates the energy required for checkpointing a byte of data into nonvolatile memory (NVM) and $N_{\text {bytes }}$ refers to the total number of bytes that are to be copied into the non-volatile memory for the checkpointing operation. The value of $E_{\text {byte }}$ depends on the kind of NVM technology in use (e.g., flash, FRAM, MRAM, etc.). On the other hand, $N_{\text {bytes }}$ depends on the program location at which the checkpoint operation is triggered, and hence varies from one checkpoint to another due to the dynamic nature of stack and heap depths, which grow and shrink during the course of program execution.

Mementos is such a checkpointing solution intended for transiently powered computers [Ransford et al. 2011; Ransford 2013]. Mementos inserts trigger points at various locations in the program such as at the end of loops, function calls, and so on, that compares the system's supply voltage to a predetermined voltage, $\mathrm{V}_{\text {TRIG }}$, as a means of tracking the remaining amount of energy. If the supply voltage is greater than $\mathrm{V}_{\text {TRIG }}$, then no action is taken and the program continues execution. Otherwise, Mementos saves a snapshot of the system state consisting of processor register values and SRAM contents (which are both volatile) onto the non-volatile flash memory for future rollback. However, Mementos is disadvantageous due to three reasons. First, it impedes program execution by checking the supply voltage proactively and repeatedly. Second, Mementos incurs a significant energy overhead by adopting flash as the NVM. Flash memory is energy intensive due to its erase and write operations, which incurs significant power and latency overheads resulting in a high $E_{\text {byte }}$. Finally, the dynamic nature of SRAM contents forces Mementos to use a conservative estimate for $N_{\text {bytes }}$, which along with the high $E_{\text {byte }}$ results in a large $\mathrm{V}_{\mathrm{TRIG}}$ of $2.6 \mathrm{~V}$. Hence, Mementos expends a significant amount of energy (Equation (1)) for the checkpoint operation. 
Our previous work, QuickRecall [Jayakumar et al. 2014c, 2015] addresses the issues with Mementos and reduces the energy overhead for checkpointing. QuickRecall utilizes FRAM, an emerging NVM, in a unified memory architecture that maps all the program sections, that is, stack, heap, text, and so on, onto the same memory technology. Traditionally, the text section is mapped onto the non-volatile ROM whilst the stack and heap sections are mapped to the volatile SRAM, which necessitates a checkpoint from RAM to NVM on power loss. QuickRecall's unified memory architecture enables in situ checkpointing in the NVM, thereby alleviating the need for copying data from the SRAM to NVM and, thus, reducing $N_{\text {bytes }}$. By adopting FRAM as the NVM, QuickRecall reduces $E_{\text {byte }}$ as well, thus reducing $E_{\text {ckpt }}$ and therefore is able to have a very low trigger voltage of $2.03 \mathrm{~V}$ (which is only $30 \mathrm{mV}$ above the minimum operating voltage of the MCU used). Additionally, QuickRecall does not impede program execution by periodically checking the supply voltage and instead saves the snapshot only once when power loss is imminent (as discussed in Section 3). Hibernus [Balsamo et al. 2015; Rodriguez Arreola et al. 2015] also utilizes FRAM as the NVM instead of flash but maps the program sections in a traditional manner, i.e., text section to FRAM and data and stack sections to SRAM, resulting in a conservative $V_{\text {TRIG }}$ that results in wasted energy for smaller checkpoint sizes. This article builds on QuickRecall by proposing to dynamically map frequently executed computational kernels and associated data to the SRAM, resulting in performance and energy benefits. Hibernus++ [Balsamo et al. 2016] proposes a scheme that sets the different system values according to the nature of the ambient energy harvesting source to minimize energy wastage. Cassens et al. [2016] also implement an interrupt-based intermittently powered systems, similar to QuickRecall and Hibernus. Bhatti and Mottola [2016] propose an incremental checkpointing scheme for intermittently powered systems. Finally, this work is an extension of our previous work in Jayakumar et al. [2016a].

\subsection{Non-Volatile Processors for Checkpointing}

An orthogonal approach for retaining state in IoT systems with an unreliable power supply is to perform the checkpoint operation entirely in hardware using non-volatile processors. These processors are designed using memory elements (flip-flops and RAM) that are augmented with a non-volatile storage to automatically checkpoint the volatile state on power loss and restore it on subsequent power availability without any software support. The progress in this branch of hardware-based checkpointing research can be chronologically arranged as simulation studies of processors with nanomagnetic devices [Kothari and Carter 2007] and floating gate elements [Yu et al. 2011], fabrication of microcontrollers with ferroelectric elements [Wang et al. 2012; Bartling et al. 2013; Khanna et al. 2014; Singhal et al. 2015], MRAM [Sakimura et al. 2014], and architectural simulation with FRAM [Ma et al. 2015a, 2015b] and Spin Transfer Torque (STT)-MRAM [Onizawa et al. 2015]. More recent research in this field includes a $100 \mathrm{MHz}$ non-volatile processor that utilizes non-volatility provided by ReRAM [Liu et al. 2016]. Non-volatile processors reduce the overhead in storing and recalling state as no instructions are required to be executed for data transfer. However, at the time of composing this article, none of the above solutions are commercially available off the shelf. Therefore, in this work, we utilize the MSP430FR5739 MCU that is embedded with FRAM and based on Texas Instruments' MSP430 architecture similarly to Zwerg et al. [2011] and Baumann et al. [2013].

\section{MOTIVATION}

In this section, we first describe the architecture, operation, and execution flow of the IoT edge devices considered in this article. Then we present an example to motivate our proposed energy-aware memory-mapping technique. 


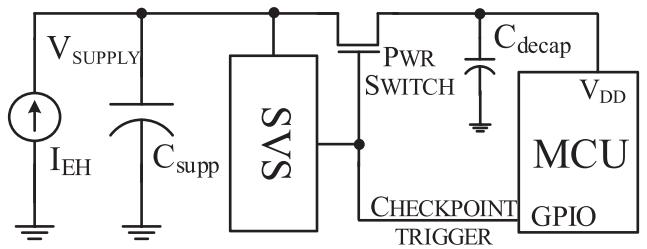

(a) Power supply architecture of an energy harvesting IoT device

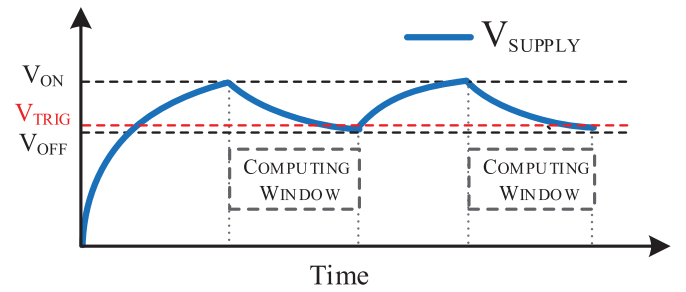

(b) Conceptual voltage profile

Fig. 1. Characteristics of edge devices considered in this work.

\subsection{Edge Device Architecture}

As mentioned in Section 2, an important feature of IoT edge devices operating with an unreliable power supply is the ability to detect and respond to interruptions in their power supply. Figure 1(a) shows the power supply architecture of an IoT device considered in this article, and Figure 1(b) illustrates a conceptual supply voltage profile that shows the typical operating stages of the IoT device with intermittent power supply. The power supply architecture consists of an ambient source, which is modeled as a current source $\left(\mathrm{I}_{\mathrm{EH}}\right)$, a supply capacitor $\left(\mathrm{C}_{\text {supp }}\right)$, a supply voltage supervisor (SVS), and a power switch. The SVS monitors the voltage $\left(\mathrm{V}_{\text {SUPPLY }}\right)$ across $\mathrm{C}_{\text {supp }}$ and has two trigger voltages, namely $\mathrm{V}_{\mathrm{ON}}$ and $\mathrm{V}_{\mathrm{TRIG}}$, to control the power switch. As shown in Figure $1(\mathrm{~b}), \mathrm{C}_{\text {supp }}$ gets slowly charged until $\mathrm{V}_{\text {SUPPLY }}$ becomes equal to $\mathrm{V}_{\mathrm{ON}}$, at which point the SVS closes (switches on) the power switch, thus connecting $\mathrm{C}_{\text {supp }}$ to the target system. Subsequently, the decoupling capacitor $\left(\mathrm{C}_{\text {decap }}\right)$ gets charged and the MCU powers on to perform the relevant computations. This is depicted as the computing window in Figure 1(b). An important characteristic of the devices that are considered in this article is that $I_{E H} \ll I_{\text {system }}$. Therefore, the system voltage continues to drop rapidly as execution progresses until $\mathrm{C}_{\text {supp }}$ discharges to the trigger voltage $\left(\mathrm{V}_{\mathrm{TRIG}}\right)$. When $\mathrm{V}_{\text {SUPPLY }}$ drops to $\mathrm{V}_{\text {TRIG }}$, the SVS transitions its output signal from high to low, thus opening the power switch and disconnecting $\mathrm{C}_{\text {supp }}$ from the target system to prevent further loss in energy. Additionally, the same signal is also used to trigger a checkpoint of the system state by the MCU. The energy required for checkpointing is derived from $\mathrm{C}_{\text {decap }}$ as is given by the following equation:

$$
E_{\text {ckpt }}=\frac{1}{2} C_{\text {decap }}\left(V_{T R I G}^{2}-V_{O F F}^{2}\right),
$$

where $\mathrm{V}_{\mathrm{OFF}}$ corresponds to the voltage at which the MCU powers off. Note that enough margin is built into $V_{\text {TRIG }}$ to satisfy a successful checkpoint. ${ }^{1}$ The process repeats with $\mathrm{C}_{\text {supp }}$ getting recharged from the energy harvesting source. This implies that at the beginning of every power cycle, the system is guaranteed an energy supply of $\frac{1}{2} \mathrm{C}_{\text {supp }}\left(\mathrm{V}_{\mathrm{ON}}{ }^{2}-\mathrm{V}_{\text {TRIG }}{ }^{2}\right)$ agnostic to the modality of (and variations in) energy harvesting.

\subsection{Motivational Example}

Although FRAM is better than flash by having lesser write energy and lacking an explicit erase operation, it compares poorly to SRAM in terms of access latency. In an FRAM-enabled MCU, MSP430FR5739 [Texas Instruments 2014], we observed that the FRAM access latency is $3 x$ longer as compared to the on-chip SRAM. Consequently, a

\footnotetext{
${ }^{1}$ In this work, we build on QuickRecall, which uses a V VRIG set to 2.03V. When a checkpoint trigger occurs, the power switch disconnects the MCU from the supply. However, $\mathrm{C}_{\text {decap }}$ supplies rest of the energy available until $\mathrm{V}_{\mathrm{OFF}}=2 \mathrm{~V}$ to perform the checkpointing operation [Jayakumar et al. 2015].
} 


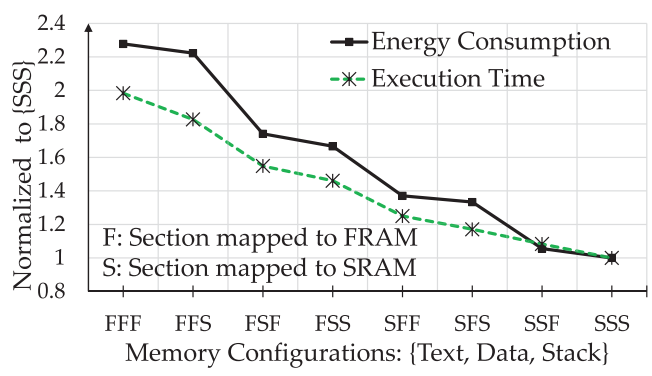

(a) $C R C-I$

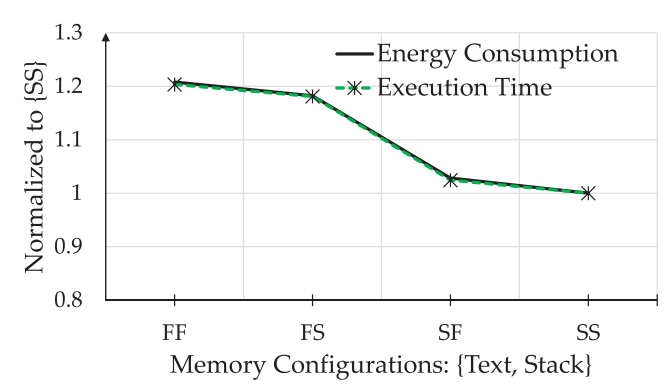

(b) CRC-II with no data section

Fig. 2. Energy consumption for the CRC functions in various memory configurations.

unified-FRAM memory architecture will result in longer execution times. To quantify the impact that memory mapping has on execution energy and time, we perform an experiment using the MSP430FR5739 MCU that consists of 1KB of volatile SRAM and $16 \mathrm{~KB}$ of non-volatile FRAM.

Two cyclic redundancy checksum (CRC) functions are considered for evaluation and are described below. Both the functions compute the 16-bit CRC of 64 bytes of data. CRC-I looks up a 512-byte static table for computing the checksum and has a large memory footprint. $C R C-I$ has three different sections that are of interest, namely a text section that contains the executable code, a data section that contains the look-up table, and the stack. On the other hand, CRC-II computes CRC using polynomials and uses only the text and stack sections. For both the programs, we iteratively map each section to both FRAM and SRAM and measure the execution energy and time.

Figures 2(a) and (b) show the measured energy consumption and latency associated with each memory mapping for executing $C R C-I$ and $C R C-I I$, respectively. The memory mapping is represented in binary format with the text section as the MSB, followed by data and stack sections. An S signifies that the section is mapped onto SRAM, whereas an F indicates a mapping to the FRAM. For example, configuration \{SFS \} in Figure 2(a) signifies that the text and stack sections of CRC-I are mapped onto the SRAM and that the data section is mapped onto the FRAM. Observe that for both the programs, a unified SRAM mapping results in the least energy consumption while a unified FRAM mapping results in the maximum energy consumption. Overall, we note that for both $C R C-I$ and $C R C-I I$, any of the SRAM-mapped configurations consume less energy (by as much as 2.28x) and execute faster (by as much as $1.98 \mathrm{x}$ ) as compared to the unified FRAM configuration. However, additional data transfer operations are required to ensure system reliability and continuity of program execution across power cycles when operating in an SRAM-mapped configuration. Hence, a tradeoff exists between the data transfer cost and the execution cost. Thus, an optimal memory-map configuration may lie in between an FRAM-only approach (that has the maximum execution energy but least data transfer overhead) and an SRAM-only configuration (that has the least execution energy but maximum data transfer overhead). Our proposed solution aims to find the optimal memory-map configuration that minimizes the overall energy cost for IoT edge devices without sacrificing reliability.

\section{DESIGN}

In this section, we first describe the challenges associated with finding the energyoptimal memory configuration and then highlight the salient features of the proposed design. 


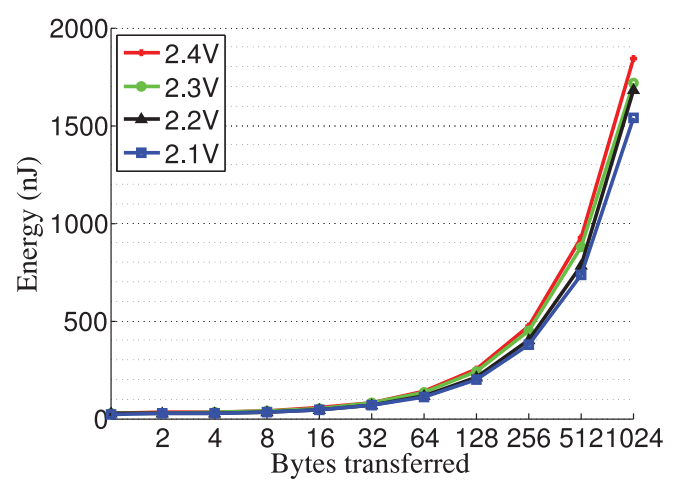

Fig. 3. Migration overhead.

\subsection{Challenges}

Determining the optimal memory-map configuration for a program is challenging due to two reasons, namely the data transfer overhead involved in moving bytes back and forth between SRAM and FRAM the diverse nature of applications. Specifically, the data transfer overhead originates from the processes of migration and checkpointing. Migration overhead is best described as the energy incurred in transferring sections from FRAM to SRAM. For example, if the considered function has the least energy consumption in configuration $\{\mathrm{SFF}\}$, the executable code that resides in the non-volatile memory initially needs to be migrated to SRAM and thus incurs a migration overhead. The number of bytes to be migrated is function dependent (e.g., CRC-II does not use the table thus having a smaller migration overhead) and application dependent (e.g., the same function may have different input datasets when called from two locations in the program). Figure 3 shows the measured energy overhead for migration as a function of the number of bytes to be migrated for supply voltages ranging from $2.1 \mathrm{~V}$ to $2.4 \mathrm{~V}$. From the graph, we observe that migration incurs $\sim 1.6 \mathrm{~nJ}$ of energy per byte of data that is transferred from FRAM to SRAM. Note that the difference in migration energy cost is negligible across the range of supply voltages used in the experiment. Therefore, migrating a section at any stage in the computing window (Figure 1(b)) incurs comparable costs.

Checkpointing, in the context of this work, is the process of saving the system state from SRAM to FRAM, that is, concerns with the data moving in the other direction. Our experiments show that the energy per byte cost of checkpointing is similar to that of migration. However, checkpoint energy is non-deterministic due to the dynamic nature of stack and heap sizes as mentioned in Section 2. This places constraint on $\mathrm{V}_{\text {TRIG }}$ as an incomplete checkpoint will result if $E_{\text {ckpt }}$ (Equation (2)) is insufficient to save a full snapshot of the system state on an imminent power loss leading to a loss or corruption of the system state. The energy spent in executing the program in such a scenario is wasted, and additional energy needs to be spent in program re-execution subsequently. Further, the system loses reliability in such scenarios. On the other hand, making an overly conservative estimate of the checkpoint energy will lead to under-utilization of the available energy and cause wastage. Therefore, a deterministic policy that accurately estimates the checkpoint energy per configuration is imperative in deciding the optimal memory map for each function.

Last, the diversity in IoT device characteristics introduces another dimension of complexity in determining the optimal configuration for the memory mapping of a function across platforms. For example, a different value for $\mathrm{C}_{\text {supp }}$ could make the $C R C-I$ function run to completion in a single power cycle in one IoT device but take 
multiple power cycles in another for the same memory-map configuration. This renders generalizing a particular configuration as an optimal memory map impossible, affecting program portability.

\subsection{Proposed Solution}

An important observation is that the applications that are run on such systems exhibit a deterministic nature in their execution flow. The typical execution flow in the IoT device involves making a sensor measurement of a physical phenomenon (e.g., temperature, humidity, etc.) by collecting a fixed number of samples, performing computations on the collected data (e.g., filtering, statistical computations such as mean, standard deviation, etc.) that take a deterministic and constant amount of clock cycles, and then transmitting it for further actuation or logging depending on the energy remaining in the system. Such a relatively simple software design results in the absence of run-torun variations in execution times, data sizes, and so on, thus making them predictable and deterministic.

However, the primary source of non-determinism in such systems spawns from the unpredictability in checkpoint size whenever the memory-map configuration includes SRAM for data allocation. The non-determinism and inefficiency associated with mapping sections to SRAM can be primarily attributed to the unpredictability in checkpoint size. It also impacts the choice of the trigger voltage as explained in Section 2. If the system receives a checkpoint trigger signal when operating in the \{SSS\} configuration, then only the stack and data sections need to be copied over to the NVM. The need for checkpointing the text section is an uncommon case as IoT applications rarely consist of self-modifying code. Therefore, the main goal of our proposed design is to reduce the non-determinism associated with the checkpoint operation and to improve the overall performance.

\subsubsection{Design Choices.}

Functions as the basic unit. Functions can be considered as quasi-independent entities in a program that are self-contained in terms of their sections having their own text, data, and stack sections that can be mapped onto memory at runtime. Moreover, a function also has the property that its stack ceases to exist on returning to its caller. Therefore, checkpointing at the end of a function at its boundary decreases the amount of data that needs to be checkpointed, which in turn reduces the non-determinism. Hence, we propose to perform checkpoint operations only at these boundaries where the checkpoint size is reduced and deterministic. Figure 4 illustrates our overall approach wherein each function $f_{0}()$ in the program is an independent entity that can be mapped to either FRAM or SRAM.

Memory architecture. Figure 4 also shows the memory architecture for a hybrid FRAM-SRAM microcontroller. The non-volatile FRAM memory is partitioned into two distinct regions, namely FRAM- $p$ and FRAM- $t$. FRAM- $p$ is the memory space where persistent data such as the text section, constants, and so on, are stored. FRAM- $t$ defines the space where a function can map temporary sections such as stack and data as dictated by the memory-map configuration during function execution, that is, FRAM- $t$ acts as a slower but non-volatile RAM. Note that no section is mapped to the SRAM initially. As the program executes, different functions can dynamically allocate sections onto the SRAM. Since each function is handled as an independent entity, sections that are mapped onto the SRAM become invalid once the function runs to completion. The text section has a fixed size and therefore occupies one end of the address space. On the other hand, the stack section grows and shrinks during the course of function-execution and hence occupies the other end of the SRAM address space. The data section occupies the address space adjacent to the text section in 


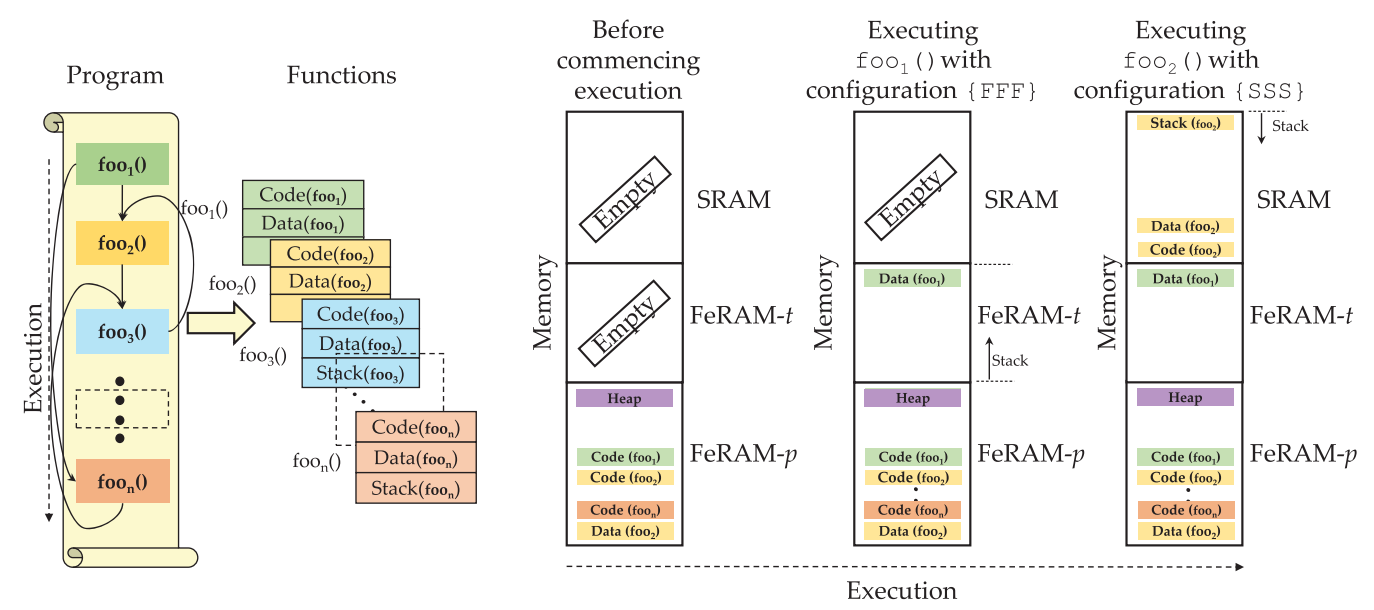

Fig. 4. Memory mapping for hybrid FRAM-SRAM MCUs.

SRAM. Note that in spite of such an arrangement, the sections may still collide during execution depending on the total SRAM capacity and section sizes. In such a case, the memory-map configuration is invalid (and disallowed), as the function can never complete correctly in this configuration. The data section inside a program can be perceived to be consisting of global variables, constants, heap, and so on. In this work, we refer to the global variables, arrays, and constants as the data section that can be migrated between the SRAM and FRAM. The heap section, which is set aside for dynamic memory allocation, is statically mapped onto the FRAM. The reasons governing such a design decision are twofold. First, in most embedded systems, the maximum size of the stack and heap sections are pre-defined by the programmer according to the SRAM capacity. However, as we propose to migrate additional sections to SRAM, migrating the entire heap section will increase the chance of a collision in the SRAM and is better avoided. Additionally, copying the entire heap might be a futile exercise as the heap may not be completely utilized. Second, copying the active part of the heap is challenging as it requires keeping track of the size of the heap. The heap may be partially filled and fragmented, which makes tracking the active locations even more cumbersome. Hence, in this work, the (single) heap is mapped exclusively to the FRAM.

\subsubsection{Design Methodology.}

Energy-aware memory mapping. Arriving at the optimal memory map for a particular function requires that the energy consumption for performing the processes of migration, execution, and checkpointing be considered together. The optimal memory map for a function is one that can perform the three processes within a single power cycle with the least amount of energy. However, since the amount of input energy is dependent on the system implementation, all memory-map configurations may not be possible for a function. This is because in certain memory-map configurations, the energy required for migration, execution, and checkpointing may exceed the input energy per power cycle. In fact, functions may exist that cannot complete within a single power cycle for any configuration. Therefore, finding the optimal memory-map configuration for a function has to be performed in an energy-aware manner. We propose $e M$-map as a one-time characterization step that arrives at the optimal memory map of constituent functions of a program in an energy-aware manner. Additionally, we propose to execute $e M$-map only after deployment to ensure that the resultant memory 


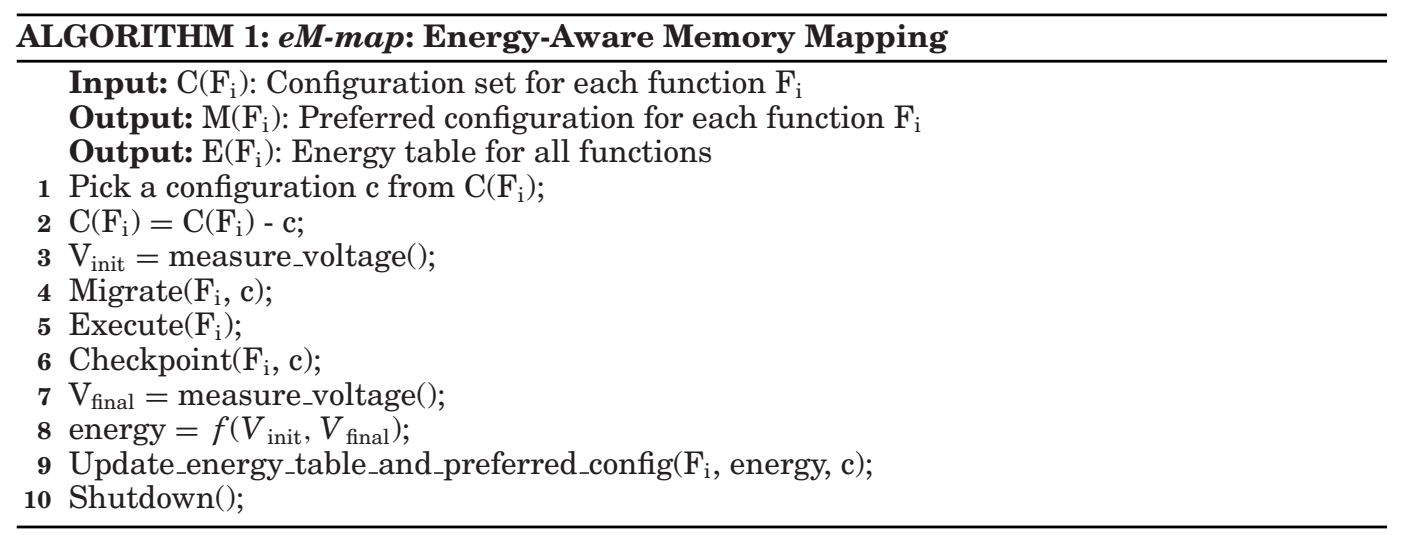

map is energy optimized for the particular IoT edge device, thus making eM-map a portable solution. A brief description of the $e M$-map algorithm (Algorithm 1) follows.

$e M$-map successively iterates through all possible configurations for a function to arrive at the energy-optimal configuration. The default memory-map assignment is set to be configuration $\{F F F\}$, which corresponds to the unified FRAM configuration. In cases wherein the function cannot complete in a single power cycle for any memory-map configuration, for the sake of reliability, $e M$-map chooses the unified FRAM configuration even though it might not be energy optimal. Each iteration begins with the supply capacitor charged to $\mathrm{V}_{\mathrm{ON}}$. A memory map is assigned to the function, and $e M$-map performs the processes of migration, execution, and checkpointing and measures the cumulative energy consumed for all three stages. A memory map is considered valid only if the function successfully completes execution in that power cycle. At the end of each iteration, $e M$-map updates a table with the minimum energy configuration for the considered function. A score (henceforth referred to as energy score) is calculated by function $f$ in line 8 of Algorithm 1. This score is proportional to the energy consumption and is computed as $V_{\text {init }}^{2}-V_{\text {final }}^{2}$, where $V_{\text {init }}$ and $V_{\text {final }}$ are indicative of the initial and residual energy for the function being characterized and measured as voltages in lines 3 and 7 of the algorithm. Note that the score is independent of the capacitance, which is an invariant system parameter across iterations. Additionally, calculating the score independent of the system capacitance makes the algorithm portable across IoT devices. This score is used by $e M$-map to compare and select the energy-optimal configuration. Further, the score is also saved in the energy table to be used for future comparisons and runtime calculations. However, if all the configurations for a function become invalid, then no score can be computed and $e M$-map selects $\{\mathrm{FFF}\}$ as the memory configuration and denotes it in the energy table. The configuration stored in the table is then used at runtime for allocating sections to SRAM and FRAM, while the accompanying energy score is used as a metric to govern whether a function is executable in a power cycle. Thus, by performing the characterization once for a device, at the granularity of functions and only a single configuration per power cycle, $e M-m a p$ is able to find the optimal memory map regardless of the non-deterministic nature of the data transfer overheads and agnostic to the system parameters.

Energy-Align. Energy-Align is a runtime technique that improves the energy efficiency of IoT devices that intrinsically initiates a system shutdown in an effort to reduce the charging interval in between power cycles. Algorithm 2 describes EnergyAlign in detail. The key concept of Energy-Align is that it allows the execution of a function only if the system has sufficient energy to complete it. If Energy-Align finds 


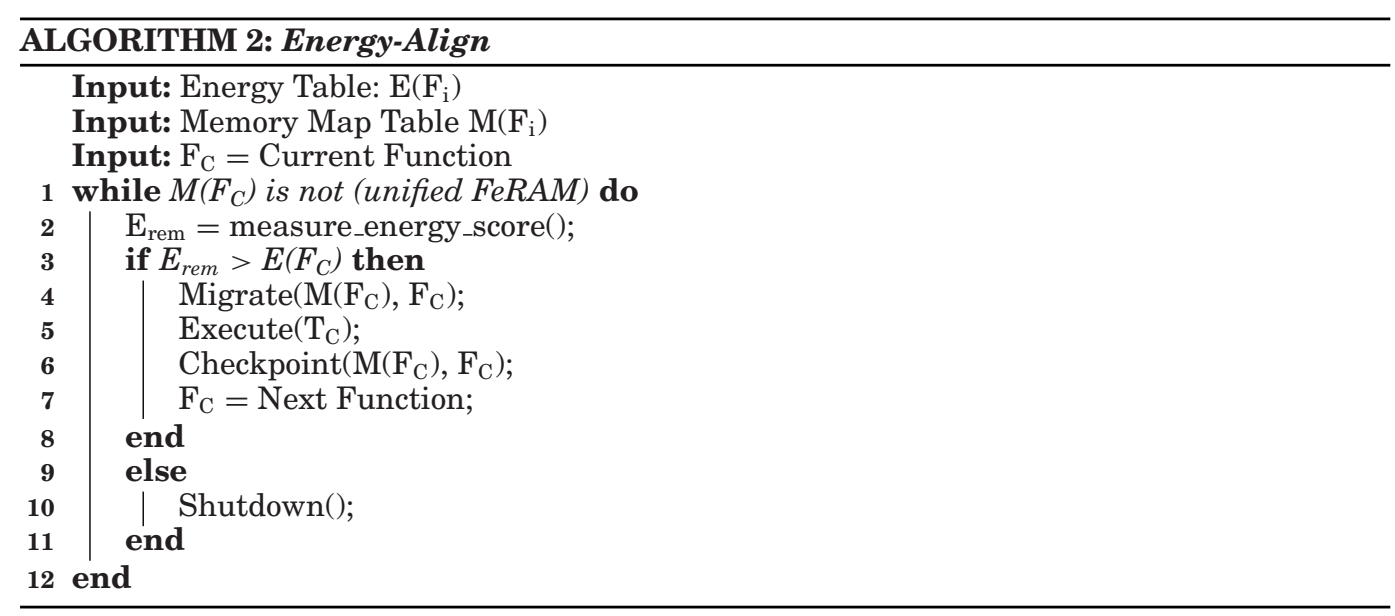

that the energy remaining is insufficient, then it performs a proactive shutdown of the system so it can recharge until $\mathrm{V}_{\mathrm{ON}}$ is faster. The characterization information from eM-map is used to predict whether the function to be executed can be successfully completed in the current power cycle. Such an approach facilitates in reducing the energy consumption in two ways. First, Energy-Align ensures that migration, execution, and checkpointing of the function happens atomically. Thus, by avoiding the partial execution of functions, Energy-Align is able to reduce the unpredictability in checkpoint sizes, thereby avoiding energy-inefficient worst-case $V_{\text {TRIG }}$ design. Hence, in our design, we are able to keep the trigger voltage at $2.03 \mathrm{~V}$, which is the same as that of QuickRecall. By construction, Energy-Align will get triggered for a checkpoint at this voltage only if it runs the function in configuration $\{F F F\}$. For all other configurations, checkpointing happens at function boundaries and, by design, the atomic operation will not extend beyond the $V_{\text {TRIG }}$ voltage. Second, by powering the system off early, Energy-Align reduces the charging time for the supply capacitor to charge back up to $\mathrm{V}_{\mathrm{ON}}$. Thus, Energy-Align executes the function in an energy-aware manner.

Figure 5 shows the benefits of Energy-Align (Figure 5(c)) over QuickRecall (Figure 5(a)) and a lazy checkpointing system (Figure 5(b); henceforth referred to as Lazy-ckpt). The lower portion of each figure depicts the supply voltage and the top portion shows the functions $F_{1}$ through $F_{3}$ executing across power cycles. Note that the charging cycle is compressed for representation. Lazy-ckpt is assumed to have an optimal memory configuration, albeit without the capability to shut down the system to perform energy alignment. Hence, Lazy-ckpt has equal execution time as Energy-Align but incurs a significant overhead due to the conservative trigger voltage setting required for guaranteeing a successful checkpoint. As depicted in Figure 5(c), Energy-Align and Lazy-ckpt run faster than QuickRecall. Additionally, note that for Energy-Align, functions are not split across power cycles, and hence Energy-Align seldom discharges the capacitor until $\mathrm{V}_{\mathrm{OFF}}$. When the system realizes it does not have sufficient energy for executing $F_{3}$, it shuts off. Since the energy consumed by Energy-Align in a power cycle is less than the total energy available (i.e., $E_{1}, E_{2}<E_{i n}$ ), it results in the system having a shorter capacitor charging time. Thus, Energy-Align improves the performance and reduces the overall energy consumption of the IoT device as compared to both Lazy-ckpt and QuickRecall.

To implement Energy-Align, we modified the architecture of the edge device as shown in Figure 6. $P$ connects $\mathrm{C}_{\text {supp }}$ to the SVS input in the default scenario. The SVS output controls the power switch that toggles the MCU between the ON and OFF states. When 


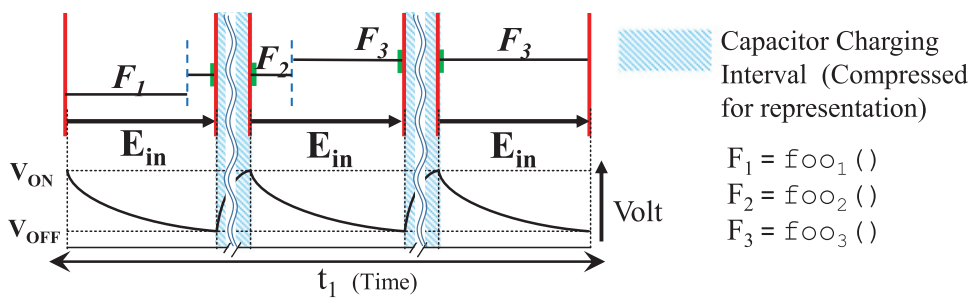

(a) Quickrecall

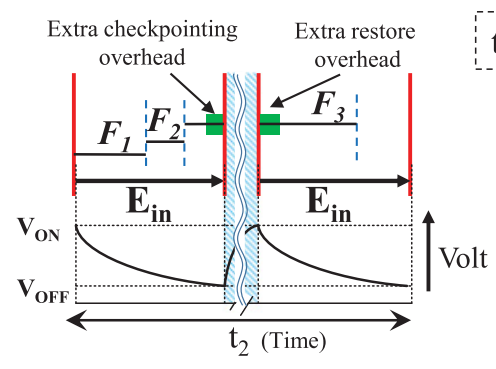

(b) Lazy Checkpointing

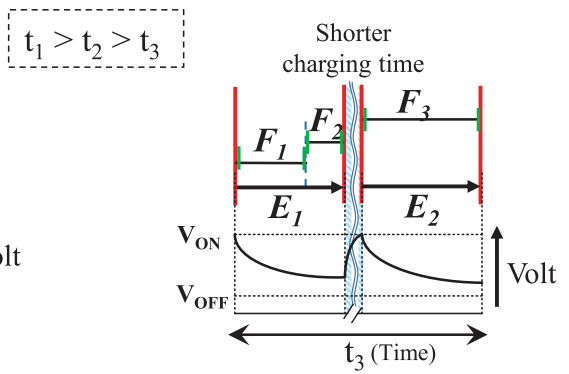

(c) Energy-align

Fig. 5. Illustration of function-execution across power cycles for QuickRecall, Lazy-ckpt, and Energy-Align.

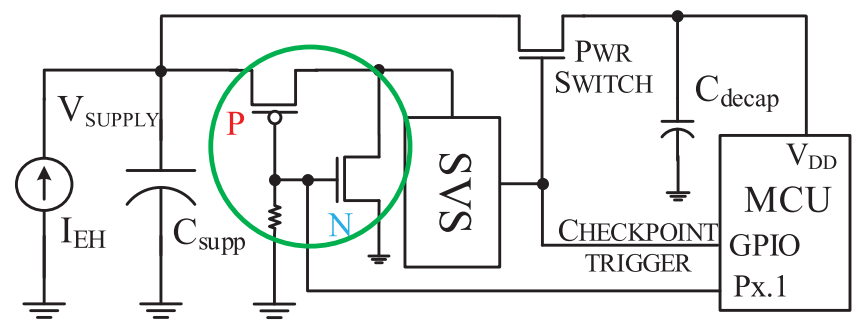

Fig. 6. Modified architecture for implementing Energy-Align.

Energy-Align is to be performed, the MCU pulls $P x .1$ to logical high, thus momentarily connecting the SVS input to ground through $N$, and disconnects $P$, the path from $\mathrm{C}_{\text {supp }}$ to the SVS input. The path through $P$ is disconnected to isolate $\mathrm{C}_{\text {supp }}$ from the ground through $N$ and avoid unwanted discharge. Since the SVS input is grounded, the power switch opens and disconnects the MCU from $\mathrm{C}_{\text {supp }}$, thus switching it OFF. When the MCU turns off, the pull-down resistor switches on $P$, thus connecting $\mathrm{C}_{\text {supp }}$ with the SVS input. Note that the SVS closes the power switch only when $\mathrm{V}_{\mathrm{SUPPLY}}=\mathrm{V}_{\mathrm{ON}}$. Since Energy-Align is performed when $\mathrm{V}_{\mathrm{OFF}}<\mathrm{V}_{\mathrm{SUPPLY}}<\mathrm{V}_{\mathrm{ON}}$, the power switch is not closed (turned ON) immediately by the SVS. Once $\mathrm{C}_{\text {supp }}$ charges to $\mathrm{V}_{\mathrm{ON}}$, SVS closes the power switch, thus turning ON the MCU. Last, we note that even though we utilize a commercially available MCU that embeds FRAM for evaluation purposes, our technique is equally applicable to MCUs with other emerging NVMs (such as MRAM, ReRAM, etc.).

\subsection{Handling Interrupts}

In traditional embedded systems, interrupts enable the CPU to provide immediate attention to an event of high importance or are used to notify the CPU about the status of an action initiated in the past. When an interrupt is triggered, the CPU pushes 
its context onto the stack and executes the associated interrupt service routine (ISR). Typically, the execution time of ISRs are intentionally kept short and fixed to prevent them from taking too much time on the processor and from blocking other interrupts in the system. The time-of-arrival of an interrupt is typically influenced by factors that are either external to the system (such as notification of change in the value of the physical phenomenon being sensed, initiation of communication, etc.) or internal to the system (such as timers, peripherals, software initiated interrupts, etc.). Broadly, interrupts can be classified as deterministic and non-deterministic according to their time-ofarrival. In this work, we define deterministic interrupts as those interrupts whose arrival is expected by software. These interrupts are usually either periodic timer interrupts or notification signals indicating the completion of an action. For example, analog measurements using an ADC take a few clock cycles to converge on the value corresponding to the sensed voltage. Hence, often the arrangement between the ADC and $\mathrm{CPU}$ is one wherein once the $\mathrm{ADC}$ conversion is initiated, it will interrupt the CPU only when the conversion completes, thus freeing the CPU to proceed with other computations or enter a low power mode. The ISR for such an interrupt is deterministic in execution time as well and typically involves copying the contents of an output buffer into a memory location. Such interrupts simplify energy analysis and can be subjected to $e M$-map and could be executed using Energy-Align. Therefore, they are not a subject of further discussion in this section.

However, non-deterministic interrupts are characterized by their unpredictability in time-of-arrival. Event-triggered interrupts fall into this class. An example is the use of self-powered flow-pressure sensors in crude-oil distributing pipes that trigger an interrupt when the pressure falls below a threshold, signaling a possible leak. In systems where available energy is also a limited resource in addition to CPU time, such interrupts pose a major challenge. In particular, the ISR of a non-deterministic (and hence, unexpected) interrupt takes up energy and time away from the function that it interrupts, which may result in incomplete execution of the function in that power cycle, prompting a re-execution of the function in the next power cycle. Even though Energy-Align in its current form has some inherent resilience to perturbations caused by such interrupts, we enhance the robustness of our design by devising a methodology for handling interrupts conforming to three design elements as described below

Design element 1: Interrupts supersede function execution. Therefore, an interrupt will always be serviced immediately, as any delay might lead to loss of state and result in a false execution. For example, an interrupt may be used to initiate data transfer from a sensor to the MCU, and any delay could cause the state to be missed, or, more catastrophically, a wrong state may be sensed, leading to wrong inferences.

Design element 2: An interrupt service routine inherits configurations from the function it interrupts such as memory-map configuration, peripheral state, and so on. These inherited configurations influence the ISR's power consumption and execution time. Therefore, the energy consumed by an ISR will vary from one invocation to another. For example, consider two scenarios that differ in the kind of peripherals that are active when an interrupt triggers. The first scenario is one wherein the MCU just performs computations (and has a current consumption of $300 \mu \mathrm{A}$ ) while in the second scenario, the MCU executes a function that requires the ADC and radio peripherals to be powered on (and has a current consumption of $5.1 \mathrm{~mA}$ ). The power consumption of the system in the latter case will be higher due to peripherals being powered on and, as a result, the ISR of an interrupt that triggers in the second scenario consumes more energy $(17 \times)$ as compared to the first. Actively monitoring the number of peripheral components that are powered on at any particular stage of 


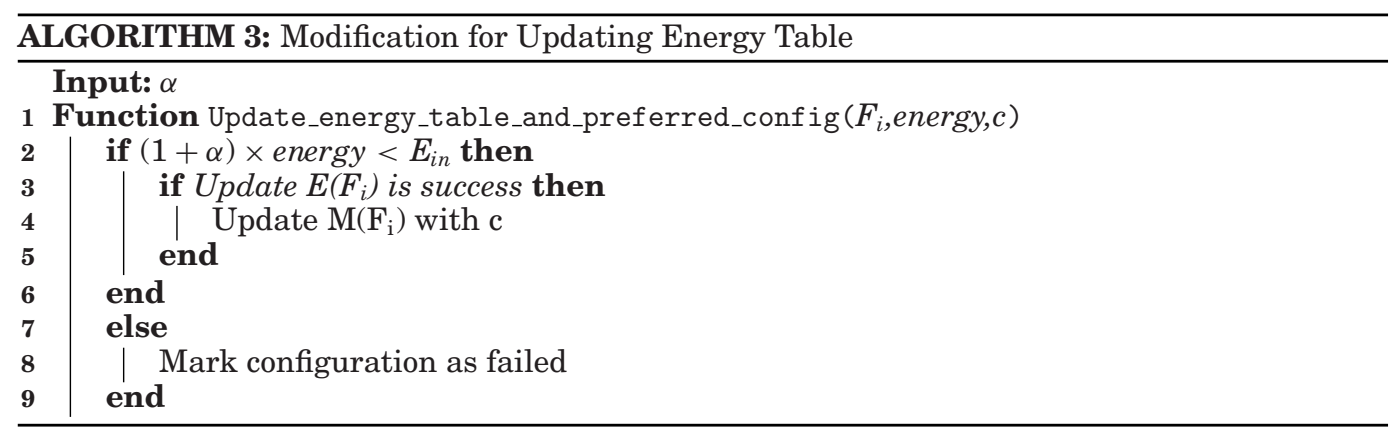

the program and controlling their power state for ISRs is detrimental to application execution and, hence, is avoided. The other configuration that the ISR inherits from the interrupted function is its memory map, albeit partially. Since ISRs are typically short pieces of code that are executed once, the text section is not migrated but executed from the non-volatile memory itself. Therefore, in our design, ISRs execute with the memory-map configuration of $\{F x y\}$ wherein $\mathrm{x}$ and $\mathrm{y}$ correspond to the memory-map configuration of the stack and data section of the function that was interrupted. Since the memory-map configuration affect the speed of execution, the mapping of the function also influences the ISR's energy consumption and varies from function to function. Hence, we do not characterize the energy consumption of an ISR and execute the ISR with the configurations inherited from the interrupted function.

Design element 3: To account for the energy required to service non-deterministic interrupts (NDIs), we allot additional energy per function as a guard-band in excess of its $e M$-map measured energy consumption. The additional amount of energy is calculated as a percentage of the function's energy consumption and is equal to $\alpha E\left(F_{C}\right)$, where $E\left(F_{C}\right)$ corresponds to the energy consumption for the function as recorded in the energy table by $e M$-map and $\alpha$ corresponds to a programmer-configurable fudge factor that determines the additional percentage of energy. We modify Energy-Align such that it compares $E_{r e m}$, which is the energy remaining in the system, with $(1+\alpha) E\left(F_{C}\right)$ in line 3 of Algorithm 2 and also modify the function that updates the energy table to reflect the effect of $\alpha$ on the memory-map configuration as shown in Algorithm 3.

Since the term $\alpha E\left(F_{C}\right)$ corresponds to the additional energy allotted for NDIs at the beginning of each function, Energy-Align will determine whether the (subsequent) function is to be executed by comparing $E_{\text {rem }}$ with $(1+\alpha) E\left(F_{C}\right)$. If $E_{\text {rem }}$ is found to be insufficient $\left(E_{\text {rem }}<(1+\alpha) E\left(F_{C}\right)\right)$, then Energy-Align will shut down the system and defer execution of the function to the subsequent power cycle. This ensures that the system will have $\alpha E\left(F_{C}\right)$ amount of energy to service NDIs in addition to that required by the function to execute successfully. The choice of value for the fudge factor $\alpha$ is system dependent and therefore is beyond the scope of this work. Although, as a rule of thumb, more NDIs in the system would mean that $\alpha$ should be set to a larger value. However, setting $\alpha$ to a value that is too large would impede program execution as more energy is buffered for NDIs, and Energy-Align will defer functions with more regularity. On the contrary, setting $\alpha$ to a value that is too low reduces the number of NDIs that can be tolerated during the execution of a particular function. Hence, a small value for $\alpha$ increases the risk of incomplete execution of a function in the presence of multiple NDIs, which will result in re-execution of the function in the following power cycle. Last, if the function completes without using (some or all of) the energy, Energy-Align would automatically add the surplus amount to the next function during the process of measuring $E_{\text {rem }}$. 


\subsection{Discussion: Design Tradeoffs}

As mentioned in Section 3.1, the applications considered in this work exhibit a deterministic nature in their execution flow. The typical execution flow of these devices involve sensing, followed by a limited set of computations before transmitting the data for further actuation or logging. Hence, they have fixed execution profiles across invocations. Extending the approach to applications with non-deterministic and dynamic execution profiles requires a modification to the way in which Algorithm 1 is performed. This is because the worst-case execution time of the function needs to be accounted for deciding the memory map. One approach would be to combine well-studied worst-case execution time analysis techniques [Wilhelm et al. 2008] for such applications with the creation of the energy table. While the approach will considerably increase application execution safety, it would allot more energy than required for the average case and, hence, is a tradeoff.

Another aspect of the proposed design is that the number of NDIs that are provisioned by it hinges on the system designer's ability to set $\alpha$. In the case where $\alpha$ is set low, there is a possible case wherein NDIs drain the remaining system energy, thus leaving the function (it interrupted) with an insufficient amount of energy to complete. Preventing such a scenario requires some form of state retention prior to the power loss. Two approaches might be considered. The first one is to execute exclusively in an FRAM-only configuration (similar to QuickRecall). However, as seen earlier (in Figures 2(a) and (b)), this approach is energy inefficient. The other approach is to make a decision during the course of execution to migrate and execute in an FRAM-only configuration. However, a tradeoff exists between executing in an FRAM-only memory configuration for safety and energy efficiency. To execute in an FRAM-only scenario, a decision must be made by the system to checkpoint the SRAM contents (if any) to FRAM. This operation must be triggered on reaching a critical energy level (which we defined as the trigger voltage). As mentioned in Section 3.1, the choice of $V_{\text {TRIG }}$ depends on the checkpoint energy, which depends on the amount of data that needs to be checkpointed; and, as mentioned earlier, the checkpoint size is unpredictable, as it depends on the program location at which the power loss happens. Hence, the $\mathrm{V}_{\text {TRIG }}$ would have to be set according to the worst-case checkpoint size of the program in addition to having a buffer for NDIs (for safety). This conservative setting of $\mathrm{V}_{\text {TRIG }}$ will ensure safety by moving into an FRAM-only configuration earlier (before the power loss) at the expense of additional checkpoint overhead and execution cost.

\section{EXPERIMENTAL RESULTS}

This section describes the experimental setup, evaluation benchmarks, and the results obtained.

\subsection{Experimental Setup}

Figure 7 shows our experimental platform and measurement setup. The Texas Instruments MSP430FR5739 [Texas Instruments 2014] MCU with 16KB of FRAM and $1 \mathrm{~KB}$ of SRAM is employed as the MCU. All the experiments are run with the MCU frequency set at $24 \mathrm{MHz}$. An FRAM access takes three clock cycles as compared to a single cycle access for SRAM. Even though the MCU has an internal SVS, we employ an external SVS to control the power switch and set $V_{\text {ON }}$ and $V_{\text {TRIG }}$. The $V_{\text {ON }}$ and $V_{\text {TRIG }}$ voltages are set to $2.3 \mathrm{~V}$ and $2.03 \mathrm{~V}$, respectively. $\mathrm{C}_{\text {supp }}$ is $330 \mu \mathrm{F}$ for initial experiments. A Tektronix 6430 Keithley source meter is used as the current supply, which acts as the energy harvesting module. The $\mathrm{I}_{\mathrm{EH}}$ is set to $400 \mu \mathrm{A}$ for all our experiments. Finally, all the latency overheads are recorded using a Tektronix MDO4104-3 oscilloscope. 


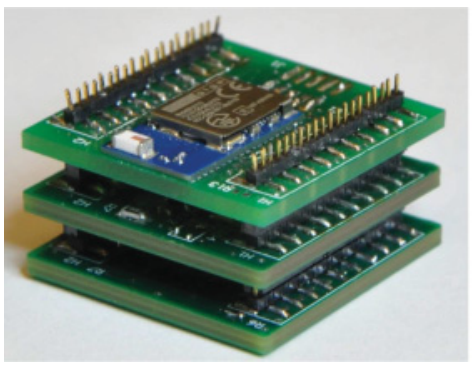

(a) QUBE Experimental Platform

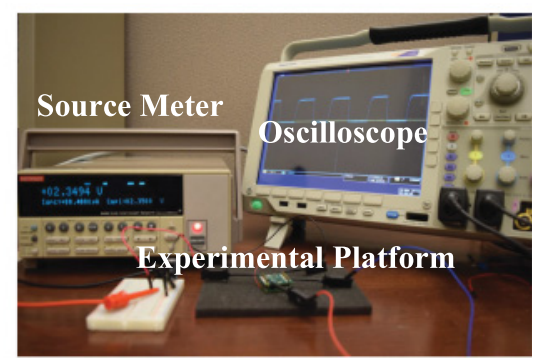

(b) Measurement Setup

Fig. 7. Experimental setup.

Table I. Evaluation Benchmarks

\begin{tabular}{|l|l|}
\hline SnC & Sample sensor readings and perform computations \\
\hline$F F T$ & $\begin{array}{l}\text { FFT(): Perform FFT on sampled data } \\
\text { Sort (): Perform bit-reversal sorting for FFT }\end{array}$ \\
\hline$C R C$ & CRC(): Compute 16-bit CRC for error detection \\
\hline$R S A$ & RSA(): Encryption algorithm \\
\hline$A E S$ & $\begin{array}{l}\text { AES algorithm made up of 4 functions, namely, addKey(), } \\
\text { shiftRows (), mixColumns (), and computeKey() }\end{array}$ \\
\hline$M M u l$ & matrixMultiply(): Perform matrix mult. on sensor data \\
\hline
\end{tabular}

\subsection{Evaluation Benchmarks}

For evaluation, we consider six different applications (shown in Table I) that are commonly used in IoT devices. As we mentioned in Section 3, all the applications are deterministic and do not vary in their execution times or input data sizes. Sense and Compute $(\mathrm{SnC})$ utilizes interrupts from the $\mathrm{ADC}$ for sampling. The interrupts are deterministic in latency and time-of-arrival and hence cause no run-to-run variation. All the functions are implemented on the device with a modified boot-loader, with software flow similar to QuickRecall, and a task manager that performs migration, execution, and checkpointing as required.

\subsection{Results}

Figure 8 shows the energy-rank ordering of different configurations for functions in the benchmark programs. The $x$-axis shows the different ranks from best to worst, while the $y$-axis shows the possible memory-map configurations. The configurations are represented in binary format as discussed in Section 3. The configurations corresponding to rank 1 denote the optimal configurations for executing the function. These are output by eM-map algorithm and used by Energy-Align. Observe that, among all the different functions plotted in Figure 8, only matrixMultiply() has the preferred configuration to be all SRAM. This means that, for most functions, the data transfer overhead of migrating all the sections to SRAM is not amortized by the reduction in energy consumption achieved by executing from SRAM, resulting in an optimal memory-map configuration that lies between $\{\mathrm{FFF}\}$ and $\{\mathrm{SSS}\}$. Additionally, note that the optimal configuration for all seven functions have the stack section to be mapped onto SRAM. This is due to the fact that the number of memory accesses to the stack is often high during the course of program execution and therefore mapping the stack section to SRAM has a significant impact on performance and energy consumption. For most functions, we observe that migrating the stack as well as just one more section of either data or text to SRAM provides the maximum energy benefits. The execution characteristics 


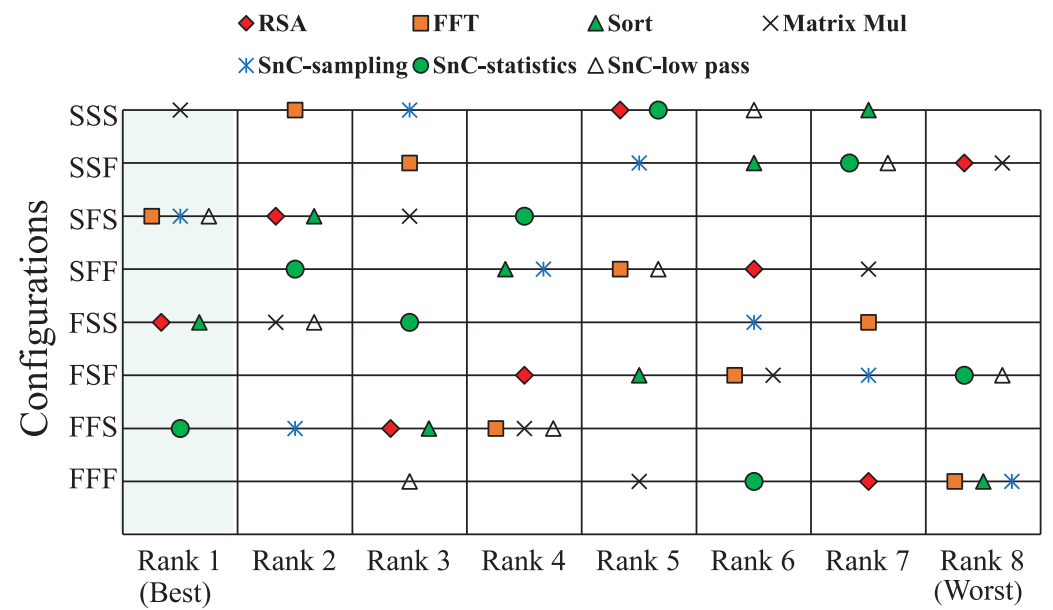

Fig. 8. Rank ordering of different memory-map configurations.

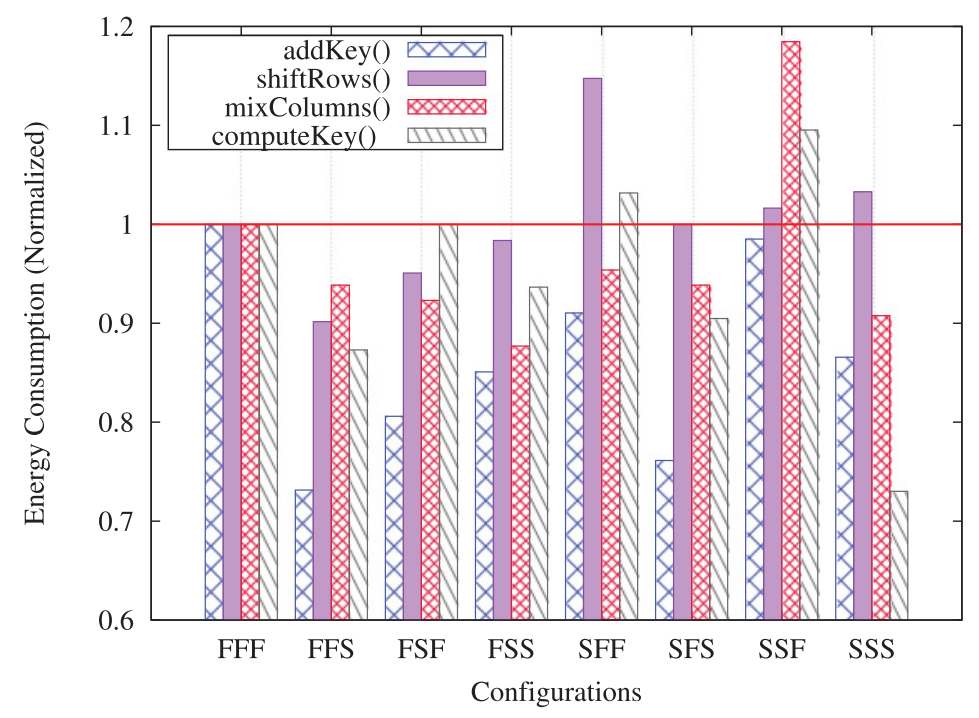

Fig. 9. Normalized energy consumption of different function configurations for AES.

and memory access pattern of the function have a bearing on the optimal memory configuration. This is ascertained by the fact that SnC-Sampling() and SnC-LowPass() have different ranks for all eight configurations in spite of having similar sizes for text and data sections. This is primarily due to the fact that SnC-LowPass() is more stack and data intensive as compared to SnC-Sampling(). Note that SnC-Sampling() involves deterministic interrupts from the $\mathrm{ADC}$, and the energy and latency overhead for the same is accounted for during characterization by $e M-m a p$.

Figure 9 shows the normalized energy consumption of all the configurations for the functions in the AES application. The energy consumption is normalized to configuration $\{F F F\}$, which corresponds to QuickRecall. Note that for some functions, migration and checkpointing of sections actually result in additional energy being expended than in the $\{F F F\}$ case. For example, even though the shiftRows () in 


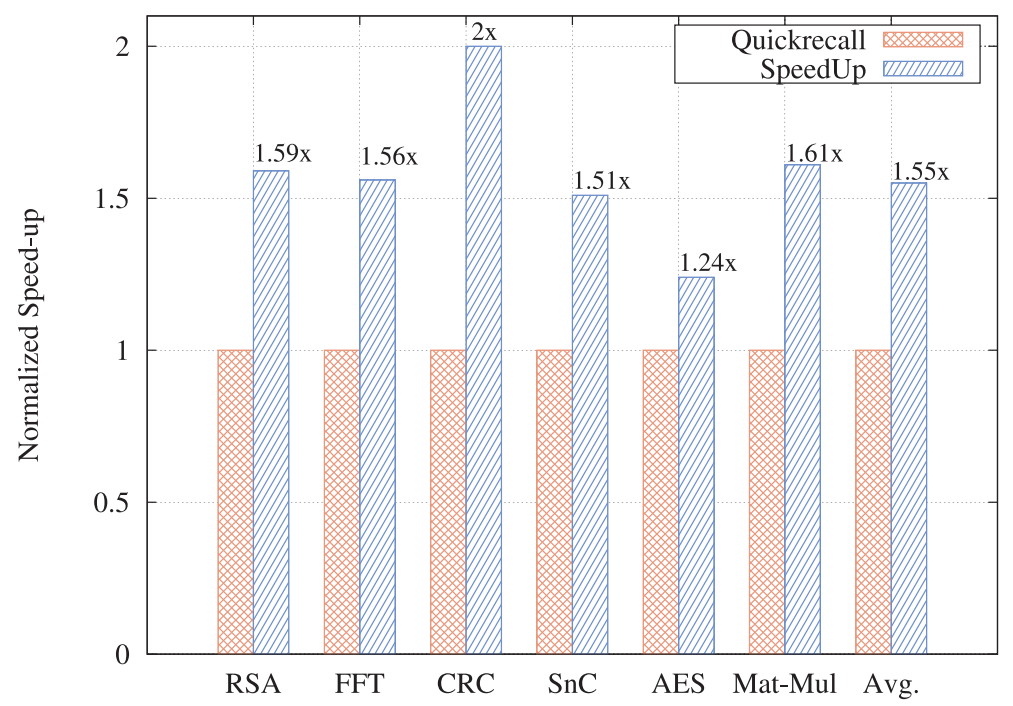

Fig. 10. Speedup comparison normalized to QuickRecall.

configuration $\{\mathrm{SFF}\}$ has only the text section to be migrated (and nothing to be checkpointed), the overall energy consumption increases. This is because shiftRows() is devoid of repetitive computational kernels such as loops, and hence the cost of migration is not amortized by the reduction in execution energy. In fact, migration of the text section involves a read of each of the bytes in the text section from FRAM, which is equivalent to executing the code once from FRAM. Therefore, migrating the code to SRAM and then executing it is wasteful. For this reason, shiftRows() has the least energy benefit in its preferred configuration among the four AES functions. Note that the optimal configuration for the functions have stack in SRAM, which concurs with our earlier observation. Overall, for the AES application, our proposed solution reduces energy consumption by $20 \%$ as compared to QuickRecall.

Energy measurement is an integral component in both eM-map and Energy-Align. This is achieved by a measurement of the supply voltage using the ADC that consumes $\leq 5 \mu \mathrm{J}$ of energy and $950 \mu \mathrm{s}$ of latency per measurement. As Figure 10 shows, this overhead is negligible as compared to the improvement in overall performance and reduction in energy consumption achieved by Energy-Align. Figure 10 shows the execution times of different IoT applications normalized to QuickRecall for a single run of the application across power cycles. The execution time includes the time required by the capacitor to regain charge and switch on the system. As is evident, the energyaware memory-mapped solution has better performance (as much as $2 \mathrm{x}$ ) as compared to QuickRecall. The speedup stems from the reduction in execution time achieved by energy-efficient memory mapping of sections by $e M$-map and also from the reduction in charging time achieved by Energy-Align. Note that even if two applications have the same overall migration overhead and optimal configurations, the unique characteristics of function-execution and memory access patterns result in different speedups for each of them.

To show that $e M$-map is agnostic to system parameters, we learn the preferred configuration for FFT-Sort () with $\mathrm{C}_{\text {supp }}$ set to $180 \mu \mathrm{F}$. Results of the experiment are shown in Table II. Most of the configurations fail to execute successfully in a single power cycle in the new system, rendering them invalid. eM-map assigns the last outstanding rank to $\{\mathrm{FFF}\}$. Finally, we note that the memory-map configuration output by $e M-m a p$ 
Table II. Rank Order of Configurations for the FFT-Sort Benchmark Using Two Different $\mathrm{C}_{\text {supply }}$ (N.V.= Not Valid)

\begin{tabular}{|c|c|c|c|c|c|c|c|c|}
\hline C supply & FFF & FFS & FSF & FSS & SFF & SFS & SSF & SSS \\
\hline \hline $330 \mu \mathrm{F}$ & 8 & 3 & 5 & 1 & 4 & 2 & 6 & 7 \\
\hline $180 \mu \mathrm{F}$ & 4 & 3 & N.V. & 1 & N.V. & 2 & N.V. & N.V. \\
\hline
\end{tabular}

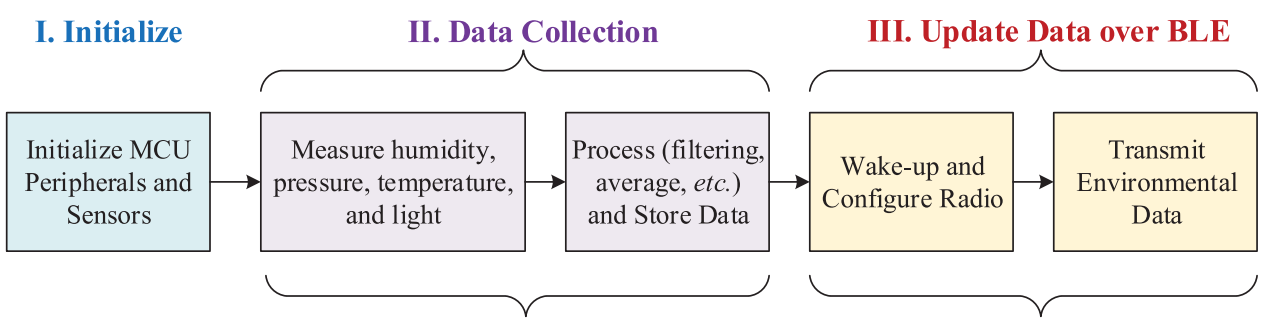

Fig. 11. Program flow used in the case study for environmental monitoring.

is agnostic to any input power trace variation. As mentioned in Section 3, the device architecture ensures the amount of available energy at the beginning of each power cycle. Any variations in the input power will only impact the amount of time the device spends in charging $\mathrm{C}_{\text {supp }}$.

\section{CASE STUDY: AN ENVIRONMENTAL MONITORING EDGE DEVICE}

In this section, we describe the case study of an IoT edge device, which executes a real application that monitors environmental conditions, such as ambient temperature, humidity, pressure, light, and so on; performs computations on them; and also transmits the data over Bluetooth Low Energy (BLE).

\subsection{Atomic Execution}

The program flow for the application is shown in Figure 11 and consists of three segments (colored differently) that are to be performed atomically, that is, without any power interruptions disrupting their execution. Atomicity is an essential criteria for applications that are to be run on intermittently powered systems for functional correctness, consistent execution, and efficient utilization of harvested energy [Lucia and Ransford 2015]. In energy harvesting systems with little notion of time, continuing executions from the saved snapshot on power restoration is not always functionally correct. This is because the checkpointed state might be inconsistent or stale and hence become invalid on wakeup. For example, during the time in which the system recovers energy to wake up again, the collected data may have already become stale and therefore should not be used for computations to follow. Similarly, power loss during communication can leave the IoT device in an inconsistent state unless proper initialization of the protocol is performed again before recommencing data transmission. For example, in systems using WiFi, the transport layer security parameters such as session ID, session ticket, encryption keys, and so on, that reside on the stack cannot be reused in a subsequent power cycle as they need to be re-configured again to prevent malicious attacks on the system. Likewise, for BLE, the link layer connection parameters that set the channel map and the seed value for the channel hop algorithm cannot be reused from a checkpointed state and need to be updated by the BLE initiator. Hence, demarcating segments within a program, and performing $e M$-map and Energy-Align at these boundaries, is vital for enabling atomicity and to make IoT edge devices energy efficient. 


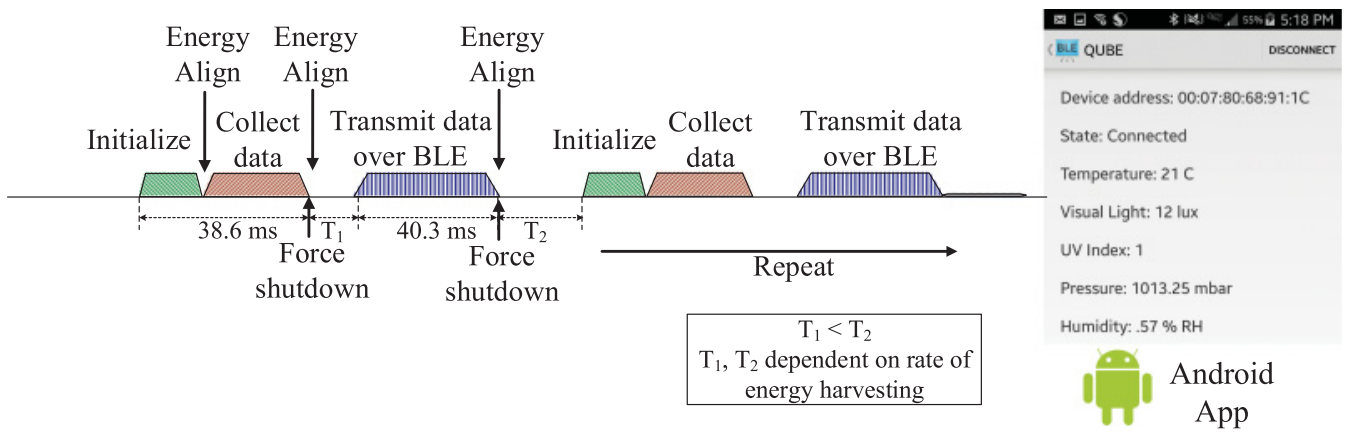

Fig. 12. Function execution time-line for the environmental monitoring application.

Consider the three segments shown in Figure 11, namely the initialization of MCU peripherals and sensors, collection of environmental data, and transmission of the sensed data over BLE. Initialization involves configuring the MCU peripherals (such as Analog to Digital Converter (ADC), Serial Peripheral Interface (SPI), Inter-Integrated Circuit (I2C), etc.) that communicate with the sensors along with configuring the sensors themselves (e.g., frequency of sampling, setting output voltage, etc.). If the initialization step is not performed in an atomic manner an a power interruption occuts, then it would result in energy being wasted, as the step needs to be repeated in the subsequent power cycle. The second segment, collecting the environmental data, involves sampling the sensor; performing computations that involve filtering, statistical calculations, and so on; and converting the sensed data into comprehensible units of measurement. Many present-day sensors communicate with the MCU over synchronous buses such as SPI and I2C. The communication in such a system is via a command-response mechanism wherein commands issued by the MCU initiates operations such as sensing, transmission, and so on, in the sensor, and the sensor responds to the MCU with data or associated messages. Losing power and checkpointing the state in between such a transaction would force the MCU into an inconsistent state on recall. This is because while the state within the MCU can be checkpointed, a checkpoint of the external sensor's state is not possible with the state of the art. Hence, appropriate commands to the sensor have to be re-issued again in the subsequent power cycle to avoid the inconsistent state. Additionally, utilizing sampled data from multiple power cycles might not be acceptable as the interval between consecutive power cycles is dependent on the unreliable energy source, which may cause the data to become stale. Hence, the step of collecting data has to be performed atomically. Finally, the last step that has to be performed in an atomic manner is the transmission of data. In addition to the aforementioned inconsistencies that occur for communication parameters across power cycles, communicating data wirelessly is an energy-intensive operation, and, therefore, waking up the radio multiple times to transmit the same set of data across multiple power cycles is an overhead best avoided.

For the case study conducted in this work, we perform Energy-Align at the segment boundaries to enforce atomic execution of each segment as shown in Figure 12. Initialization and data collection steps happen in the same power cycle atomically before the system is forcefully shutdown to gather enough energy for BLE transmission. Following this, the collected data are transmitted also in an atomic manner. The procedure is repeated as and when energy is available. To visualize the transmitted data and verify the functionality of the IoT edge device, we designed an Android mobile application and ran it on a Samsung Galaxy S5 mobile phone whose Graphical User Interface (GUI) is also shown in Figure 12. 


\subsection{Discussion: Using Non-Volatile Processors}

State-of-the-art non-volatile processors (NVPs) listed in Section 2 would partially mitigate the requirement for atomicity in the initialization step. This is because the configuration registers in NVPs are non-volatile and, therefore, will restore the checkpointed configuration in the subsequent power cycle without the need for software to reconfigure them. However, the rest of the initialization steps that include configuring the external sensors have to be performed again via a software bootup method before resuming application code execution. Similarly, a software strategy is required for NVPs to enable atomicity in application execution. Like traditional processors, NVPs also suffer from the abore-mentioned problems of stale data, false execution states, inconsistent communication states, and communication overhead arising due to program segments being broken and spread over multiple power cycles [Lucia and Ransford 2015; Colin et al. 2015]. Recent research in NVPs have tried to address the initialization overhead of peripheral registers by making them non-volatile [Li et al. 2016]. However, the inconsistency in communication and the need for re-performing the protocol's necessary hand-shaking still exists in these NVPs. Chien et al. [2016] proposed a ReRAM-based non-volatile processor to partially address the inconsistency issue. Their NVP utilizes a programmable restore point, to which the processor can wake up. In case the power interruption happens in between an input/output (I/O) communication (such as UART, SPI, etc.), utilizing the programmable restore point would roll back to the location corresponding to the beginning of data transmission. Such an architecture improves the checkpointing support from hardware while still being able to provide the programmer with the control of partitioning the program into tasks. However, the NVP allows greedy execution and therefore is susceptible to inconsistent checkpoints and energy wastage due to partial transmission. Therefore, even though NVPs help reduce the checkpoint and restore overheads, systems utilizing them would require a well-designed software architecture to enable atomicity and resolve the consistency issues in application execution. As mentioned earlier, such NVPs are not commercially available yet and, therefore, are not included in the evaluation.

\section{CONCLUSION}

In this work, we proposed a technique for energy-aware memory mapping of program sections in hybrid FRAM-SRAM MCUs used in IoT edge devices. We defined a one-time characterization technique, $e M-m a p$, that maps functions across hybrid FRAM-SRAM memory. We also proposed a technique, Energy-Align, that aligns function and power cycle boundaries, thereby achieving energy-performance benefits. Our implementation using the MSP430FR5739 MCU shows a speedup of up to $2 \mathrm{x}$ and energy reduction of $20 \%$ compared to a state-of-the-art baseline solution. Finally, we conducted a case study that showed the implementation of our techniques in the context of a real IoT application.

\section{REFERENCES}

D. Balsamo, A. Weddell, A. Das, A. Arreola, D. Brunelli, B. Al-Hashimi, G. Merrett, and L. Benini. 2016. Hibernus++: A self-calibrating and adaptive system for transiently-powered embedded devices. IEEE Trans. Comput.-Aided Des. Integr. Circuits Syst. PP, 99 (2016), 1-1.

D. Balsamo, A. S. Weddell, G. V. Merrett, B. M. Al-Hashimi, D. Brunelli, and L. Benini. 2015. Hibernus: Sustaining computation during intermittent supply for energy-harvesting systems. IEEE Embed. Syst. Lett. 7, 1 (Mar. 2015), 15-18.

S. C. Bartling, S. Khanna, M. P. Clinton, S. R. Summerfelt, J. A. Rodriguez, and H. P. McAdams. 2013. An $8 \mathrm{MHz} 75 \mu \mathrm{A} / \mathrm{MHz}$ zero-leakage non-volatile logic-based cortex-M0 MCU SoC exhibiting $100 \mathrm{VDD}=0 \mathrm{~V}$ with $<400 \mathrm{~ns}$ wakeup and sleep transitions. In Proceedings of the 2013 IEEE International Solid-State Circuits Conference Digest of Technical Papers (ISSCC). 432-433. 
A. Baumann, M. Jung, K. Huber, M. Arnold, C. Sichert, S. Schauer, and R. Brederlow. 2013. A MCU platform with embedded FRAM achieving 350nA current consumption in real-time clock mode with full state retention and $6.5 \mu \mathrm{s}$ system wakeup time. In Proceedings of the 2013 Symposium on VLSI Circuits (VLSIC). C202-C203.

N. A. Bhatti, M. H. Alizai, A. A. Syed, and L. Mottola. 2016. Energy harvesting and wireless transfer in sensor network applications: Concepts and experiences. ACM Trans. Sen. Netw. 12, 3, Article 24 (Aug. 2016), 40 pages.

N. A. Bhatti and L. Mottola. 2016. Efficient state retention for transiently-powered embedded sensing. In Proceedings of the 2016 International Conference on Embedded Wireless Systems and Networks (EWSN'16). 137-148.

B. Cassens, A. Martens, and R. Kapitza. 2016. The neverending runtime: Using new technologies for ultralow power applications with an unlimited runtime. In Proceedings of the 2016 International Conference on Embedded Wireless Systems and Networks (EWSN'16). 325-330.

T. K. Chien, L. Y. Chiou, C. C. Lee, Y. C. Chuang, S. H. Ke, S. S. Sheu, H. Y. Li, P. H. Wang, T. K. Ku, M. J. Tsai, and C. I. Wu. 2016. An energy-efficient nonvolatile microprocessor considering software-hardware interaction for energy harvesting applications. In Proceedings of the 2016 International Symposium on VLSI Design, Automation and Test (VLSI-DAT). 1-4.

A. Colin, A. P. Sample, and B. Lucia. 2015. Energy-interference-free system and toolchain support for energyharvesting devices. In Proceedings of the 2015 International Conference on Compilers, Architecture and Synthesis for Embedded Systems (CASES'15). 35-36.

H. Jayakumar, K. Lee, W. S. Lee, A. Raha, Y. Kim, and V. Raghunathan. 2014a. Powering the internet of things. In Proceedings of the 2014 International Symposium on Low Power Electronics and Design (ISLPED'14). 375-380.

H. Jayakumar, A. Raha, Y. Kim, S. Sutar, W. S. Lee, and V. Raghunathan. 2016b. Energy-efficient system design for IoT devices. In Proceedings of the 2016 21st Asia and South Pacific Design Automation Conference (ASP-DAC). 298-301.

H. Jayakumar, A. Raha, W. S. Lee, and V. Raghunathan. 2015. QuickRecall: A HW/SW approach for computing across power cycles in transiently powered computers. J. Emerg. Technol. Comput. Syst. 12, 1, Article 8 (Aug. 2015).

H. Jayakumar, A. Raha, and V. Raghunathan. 2014b. Hypnos: An ultra-low power sleep mode with SRAM data retention for embedded microcontrollers. In Proceedings of the 2014 International Conference on Hardware/Software Codesign and System Synthesis (CODES'14). Article 11.

H. Jayakumar, A. Raha, and V. Raghunathan. 2014c. QUICKRECALL: A low overhead HW/SW approach for enabling computations across power cycles in transiently powered computers. In 201427 th International Conference on VLSI Design and 2014 13th International Conference on Embedded Systems. 330-335.

H. Jayakumar, A. Raha, and V. Raghunathan. 2016a. Energy-aware memory mapping for hybrid FRAMSRAM MCUs in IoT edge devices. In Proceedings of the 2016 29th International Conference on VLSI Design and 2016 15th International Conference on Embedded Systems (VLSID). 264-269.

S. Khanna, S. C. Bartling, M. Clinton, S. Summerfelt, J. A. Rodriguez, and H. P. McAdams. 2014. An FRAMbased nonvolatile logic MCU SoC exhibiting $100 \%$ digital state retention at vdd=0 V achieving zero leakage with $<400$-ns wakeup time for ULP applications. IEEE J. Solid-State Circ. 49, 1 (Jan 2014), 95-106.

L. Kothari and N. P. Carter. 2007. Architecture of a self-checkpointing microprocessor that incorporates nanomagnetic devices. IEEE Trans. Comput. 56, 2 (Feb 2007), 161-173.

Z. Li, Y. Liu, D. Zhang, C. J. Xue, Z. Wang, X. Shi, W. Sun, J. Shu, and H. Yang. 2016. HW/SW co-design of nonvolatile IO system in energy harvesting sensor nodes for optimal data acquisition. In Proceedings of the 53rd Annual Design Automation Conference (DAC'16). Article 154.

Y. Liu, Z. Wang, A. Lee, F. Su, C. P. Lo, Z. Yuan, C. C. Lin, Q. Wei, Y. Wang, Y. C. King, C. J. Lin, P. Khalili, K. L. Wang, M. F. Chang, and H. Yang. 2016. 4.7 A 65nm ReRAM-enabled nonvolatile processor with 6x reduction in restore time and $4 \mathrm{x}$ higher clock frequency using adaptive data retention and self-writetermination nonvolatile logic. In IEEE International Solid-State Circuits Conference (ISSCC). 84-86.

C. Lu, V. Raghunathan, and K. Roy. 2010. Micro-scale energy harvesting: A system design perspective. In Proceedings of the 2010 15th Asia and South Pacific Design Automation Conference (ASP-DAC). 89-94.

C. Lu, V. Raghunathan, and K. Roy. 2011. Efficient design of micro-scale energy harvesting systems. IEEE J. Emerg. Select. Top. Circ. Syst. 1, 3 (Sep. 2011), 254-266.

B. Lucia and B. Ransford. 2015. A simpler, safer programming and execution model for intermittent systems. SIGPLAN Not. 50, 6 (Jun. 2015), 575-585.

K. Ma, X. Li, S. Li, Y. Liu, J. J. Sampson, Y. Xie, and V. Narayanan. 2015a. Nonvolatile processor architecture exploration for energy-harvesting applications. IEEE Micro 35, 5 (Sep. 2015), 32-40. 
K. Ma, Y. Zheng, S. Li, K. Swaminathan, X. Li, Y. Liu, J. Sampson, Y. Xie, and V. Narayanan. $2015 b$. Architecture exploration for ambient energy harvesting nonvolatile processors. In Proceedings of the 2015 IEEE 21st International Symposium on High Performance Computer Architecture (HPCA). 526537.

N. Onizawa, A. Mochizuki, A. Tamakoshi, and T. Hanyu. 2015. A sudden power-outage resilient nonvolatile microprocessor for immediate system recovery. In Proceedings of the 2015 IEEE/ACM International Symposium on Nanoscale Architectures (NANOARCH). 39-44.

B. Ransford. 2013. Transiently Powered Computers. Ph.D. Dissertation. University of Massachusetts Amherst.

B. Ransford, J. Sorber, and K. Fu. 2011. Mementos: System support for long-running computation on RFIDscale devices. SIGARCH Comput. Archit. News 39, 1 (Mar. 2011), 159-170.

A. Rodriguez Arreola, D. Balsamo, A. K. Das, A. S. Weddell, D. Brunelli, B. M. Al-Hashimi, and G. V. Merrett. 2015. Approaches to transient computing for energy harvesting systems: A quantitative evaluation. In Proceedings of the 3rd International Workshop on Energy Harvesting \&\#38; Energy Neutral Sensing Systems (ENSsys'15). ACM, New York, NY, 3-8. DOI : http://dx.doi.org/10.1145/2820645.2820652

N. Sakimura, Y. Tsuji, R. Nebashi, H. Honjo, A. Morioka, K. Ishihara, K. Kinoshita, S. Fukami, S. Miura, N. Kasai, T. Endoh, H. Ohno, T. Hanyu, and T. Sugibayashi. 2014. 10.5 A 90nm $20 \mathrm{MHz}$ fully nonvolatile microcontroller for standby-power-critical applications. In Proceedings of the 2014 IEEE International Solid-State Circuits Conference Digest of Technical Papers (ISSCC). 184-185. DOI:http://dx. doi.org/10.1109/ISSCC.2014.6757392

V. K. Singhal, V. Menezes, S. Chakravarthy, and M. Mehendale. 2015. 8.3 A 10.5 $\mu \mathrm{A} / \mathrm{MHz}$ at $16 \mathrm{MHz}$ singlecycle non-volatile memory access microcontroller with full state retention at $108 \mathrm{nA}$ in a $90 \mathrm{~nm}$ process. In Proceedings of the 2015 IEEE International Solid- State Circuits Conference (ISSCC). 1-3.

Texas Instruments. 2014. MSP430FR573x Mixed-Signal Microcontrollers. Retrieved from http://www.ti.com/ lit/ds/symlink/msp430fr5739.pdf. (2014).

C. Wang, N. Chang, Y. Kim, S. Park, Y. Liu, H. G. Lee, R. Luo, and H. Yang. 2014. Storage-less and converterless maximum power point tracking of photovoltaic cells for a nonvolatile microprocessor. In Proceedings of the 2014 19th Asia and South Pacific Design Automation Conference (ASP-DAC). 379-384.

Y. Wang, Y. Liu, S. Li, D. Zhang, B. Zhao, M. Chiang, Y. Yan, B. Sai, and H. Yang. 2012. A 3us wake-up time nonvolatile processor based on ferroelectric flip-flops. In Proceedings of the 2012 Proceedings of the ESSCIRC (ESSCIRC). 149-152.

R. Wilhelm, J. Engblom, A. Ermedahl, N. Holsti, S. Thesing, D. Whalley, G. Bernat, C. Ferdinand, R. Heckmann, T. Mitra, F. Mueller, I. Puaut, P. Puschner, J. Staschulat, and P. Stenström. 2008. The worst-case execution-time problem\&Mdash;overview of methods and survey of tools. ACM Trans. Embed. Comput. Syst. 7, 3, Article 36 (May 2008).

W. Yu, S. Rajwade, S. Wang, B. Lian, G. E. Suh, and E. Kan. 2011. A non-volatile microcontroller with integrated floating-gate transistors. In Proceedings of the 2011 IEEE/IFIP 41st International Conference on Dependable Systems and Networks Workshops (DSN-W). 75-80. DOI : http://dx.doi.org/10.1109/ DSNW.2011.5958839

M. Zwerg, A. Baumann, R. Kuhn, M. Arnold, R. Nerlich, M. Herzog, R. Ledwa, C. Sichert, V. Rzehak, P. Thanigai, and B. O. Eversmann. 2011. An $82 \mu \mathrm{A} / \mathrm{MHz}$ microcontroller with embedded FeRAM for energy-harvesting applications. In Proceedings of the 2011 IEEE International Solid-State Circuits Conference Digest of Technical Papers (ISSCC). 334-336.

Received December 2015; revised May 2016; accepted August 2016 